\title{
Zinc-Impregnated Mesh for Abdominal Wall Repair Reduces Infection in a Rat Model of Peritonitis
}

\author{
Yagmur Yurtkap, MD, ${ }^{a, *}$ An P. Jairam, MD, $P h D, b$ \\ Ruth Kaufmann, MD, PhD, ${ }^{a, c}$ Leonard F. Kroese, MD, PhD, ${ }^{d}$ \\ Marian C. Clahsen-van Groningen, MD, PhD, ${ }^{e}$ \\ Johan W. Mouton, MD, PhD, ${ }^{f}$ Anand G. Menon, MD, PhD, 9 \\ Gert-Jan Kleinrensink, PhD, ${ }^{h}$ Johannes Jeekel, $M D, P h D,{ }^{h}$ \\ Johan F. Lange, MD, PhD, ${ }^{a, g}$ and Eric J. Belt, $M D, P h D^{i}$ \\ ${ }^{a}$ Department of Surgery, Erasmus University Medical Center, Rotterdam, the Netherlands \\ ${ }^{\mathrm{b}}$ Department of Surgery, Catharina Ziekenhuis, Eindhoven, the Netherlands \\ ${ }^{c}$ Department of Surgery, Tergooi, Hilversum, the Netherlands \\ ${ }^{\mathrm{d}}$ Department of Surgery, Reinier de Graaf Gasthuis, Delft, the Netherlands \\ e Department of Pathology, Erasmus University Medical Center, Rotterdam, the Netherlands \\ ${ }^{\mathrm{f}}$ Department of Microbiology, Erasmus University Medical Center, Rotterdam, the Netherlands \\ ${ }^{g}$ Department of Surgery, IJsselland Ziekenhuis, Capelle aan den IJssel, the Netherlands \\ ${ }^{\mathrm{h}}$ Department of Neuroscience \& Anatomy, Erasmus University Medical Center, Rotterdam, the Netherlands \\ ${ }^{i}$ Department of Surgery, Albert Schweitzer Ziekenhuis, Dordrecht, the Netherlands
}

\section{A R T I C L E I N F O}

Article history:

Received 4 June 2019

Received in revised form

3 September 2019

Accepted 19 September 2019

Available online xxx

Keywords:

Complex hernias

Contamination

Zinc-impregnated mesh

\section{A B S T R A C T}

Background: The objective of this study was to assess whether a zinc-impregnated polypropylene mesh (ZnMesh) has better antibacterial properties in a contaminated environment compared with a regular polypropylene mesh.

Materials and methods: Thirty-eight Wistar Han rats underwent cecal ligation and puncture to induce peritonitis $24 \mathrm{~h}$ before implantation of an intraperitoneal ZnMesh or a regular polypropylene mesh. Primary outcome was the number of colony forming units (CFU) per sample (mesh and abdominal wall). Secondary outcomes were macroscopic (incorporation of mesh, abscesses, and adhesions on mesh surface) and histological (inflammatory cell reaction, mesh-specific parameters, and collagen deposition) parameters. All outcomes were evaluated after 30 and $90 \mathrm{~d}$.

Results: After $30 \mathrm{~d}$, no significant difference in CFU per sample was present between the ZnMesh and control groups. After $90 \mathrm{~d}$, a lower number of CFU per sample was present in the ZnMesh group compared with the control group (trypticase soy agar with $5 \%$ sheep blood: $0 \log _{10} \mathrm{CFU} /$ sample IQR: 0-1.40 versus $1.58 \log _{10} \mathrm{CFU} /$ sample IQR: $0-4.30, P=0.012$; MacConkey: $0 \log _{10} \mathrm{CFU} /$ sample IQR: 0-2.65 versus $1.18 \log _{10} \mathrm{CFU} / \mathrm{sample}$ IQR: 0-4.04, $P=0.438)$. After $90 \mathrm{~d}$, the percentage of adhesions on mesh surface was significantly higher in the ZnMesh group (95\% IQR: $60 \%-100 \%$ versus $50 \%$ IQR: $23 \%-75 \%, P=0.029$ ). No differences were seen in other macroscopic outcomes or histology.

\footnotetext{
* Corresponding author. Department of Surgery, Erasmus University Medical Center, Room Ee-173, Dr. Molewaterplein 40, PO BOX 2040, 3000 CA Rotterdam, the Netherlands. Tel: +31 107043683

E-mail address: y.yurtkap@erasmusmc.nl (Y. Yurtkap).

0022-4804/\$ - see front matter @ 2019 Elsevier Inc. All rights reserved.
}

https://doi.org/10.1016/j.jss.2019.09.046 
Conclusions: A significantly lower number of CFU per sample was found in the ZnMesh group after $90 \mathrm{~d}$. After $30 \mathrm{~d}$, no statistically significant differences in CFU per sample were seen. This result suggests that the ZnMesh group has better antibacterial properties in a contaminated environment. However, this is at the cost of a significantly higher percentage of adhesions.

(c) 2019 Elsevier Inc. All rights reserved.

\section{Introduction}

Prosthetic implants are used for the repair of abdominal wall hernias, and their application results in significantly lower recurrence rates. ${ }^{1}$ However, the use of a nonabsorbable synthetic mesh for hernia repair in a contaminated field remains controversial given the higher risk of postoperative infection. ${ }^{2}$ Mesh infection is one of the most severe and disastrous complications after hernia repair and may require surgical removal of the implanted scaffold. ${ }^{3}$ Mesh explantation may lead to patient morbidity, prolonged hospital admission, and increasing healthcare costs. ${ }^{4}$ Biologic implants have been promoted for contaminated fields for a long time without presenting high-level evidence. ${ }^{5}$ In a study performed by Rosen et al., ${ }^{6}$ the overall hernia recurrence was 31\% using a biological mesh in a contaminated abdominal wall defect, after a follow-up of 21.7 mo (range 1-74 mo). In addition, higher cost of biologic meshes compared with synthetic meshes is a drawback. ${ }^{7}$ Despite the wide selection of available meshes, the search for the ideal mesh to use in contaminated fields is still ongoing.

To reduce the incidence of infection, several antibacterial mesh coatings have previously been investigated. ${ }^{8,9}$ Bacterial attachment and proliferation are necessary steps in the development of an infection depending on several factors, such as the type of polymer and its structure. ${ }^{10}$ Recently, it was found that zinc ions are able to inhibit multiple activities of bacteria, for instance transmembrane proton translocation, glycolysis, and acid tolerance. ${ }^{11}$ In addition, zinc oxide may disturb metabolic pathways and exhibit an antibacterial effect on both Escherichia coli and Staphylococcus aureus. ${ }^{11}$ Until now, the polypropylene mesh incorporated with zinc ions (ZnMesh) has only been examined in in vitro models.

The primary objective of this animal study was to determine whether a polypropylene mesh incorporated with zinc ions has better antibacterial properties when placed in a contaminated environment compared with a regular polypropylene mesh. The secondary objectives were to assess ingrowth of the mesh, abscess formation, and adhesion. Furthermore, histological parameters were assessed, such as inflammatory cell response, mesh-specific parameters, and collagen deposition.

\section{Material and methods}

The study protocol was approved by the Ethical Committee on Animal Experimentation of the Erasmus University Medical Center (Rotterdam, the Netherlands, license number: AVD101002015179) and was performed in accordance with the ARRIVE guidelines on the use of laboratory animals. ${ }^{12}$

\section{Animals}

Thirty-eight male Wister Han rats, weighing 280-325 g, were purchased from Charles River Laboratories ('s-Hertogenbosch, the Netherlands). The animals were bred under specific pathogen-free conditions. All rats were housed in pairs in individually ventilated cages under $12 \mathrm{~h}$ dark/light cycles. The temperature was kept between $20^{\circ} \mathrm{C}$ and $24^{\circ} \mathrm{C}$, and relative humidity was $50 \%$ to $60 \%$ in the laboratory. Standard rat chow and water was provided ad libitum. The rats were accustomed to laboratory conditions $1 \mathrm{wk}$ before the start of the experiment.

\section{Meshes}

Regular polypropylene meshes and ZnMesh were provided by the producer (Parx Plastics, Rotterdam, the Netherlands). An existing polypropylene mesh was chemically and physically treated with dietary zinc ( $\mathrm{Zn} \mathrm{2+)}$. This treatment resulted in positive ionic surface of the polymer. Zinc ions do not migrate during time, and the ZnMesh remains biologically inert. It was hypothesized that the positive ionic surface makes the surface hostile to bacteria, reduces the capability to form biofilm, and interferes with the bacteria proliferation without releasing ions.

\section{Surgical procedure}

Preoperatively, 38 rats were randomly divided into two groups to receive either the ZnMesh $(n=20)$ or regular polypropylene mesh $(n=18)$. These two groups were again randomly divided into two groups for a follow-up of 30 or $90 \mathrm{~d}$. Experiments were done under aseptic conditions in an operation room for small animals. All rats were anesthetized with a combination of isoflurane and oxygen inhalation. Preoperatively, a single dose of $0.05 \mathrm{mg} / \mathrm{kg}$ buprenorphine was administered subcutaneously. After anesthesia, the abdominal skin was shaved, disinfected with alcohol $70 \%$, and subsequently a $3-\mathrm{cm}$ midline incision was performed, to enter the abdominal cavity.

\section{Cecal ligation puncture model}

The cecal ligation puncture model was used for the induction of peritonitis. ${ }^{13}$ On day 0 , ligation of the cecum was performed just distal to the ileocecal valve with a nonabsorbable polyamide suture (5-0 Ethilon; Ethicon, Inc., Sommerville, NJ), without interrupting the bowel continuity. Subsequently, a puncture with an 18-gauge needle was performed distally in the cecum. The fascia and skin were closed in two layers with running absorbable polyglycolic acid sutures (5-0 Safil; B. Braun, Melsungen, Germany). Postoperatively, all animals received $5 \mathrm{~mL}$ sodium chloride 0.9 per cent subcutaneously and were placed under a heating lamp to prevent 
hypothermia. After $24 \mathrm{~h}$ (day 1), all rats were anesthetized with the same inhalation mixture as on day 0 and the abdominal cavity was disinfected and reopened. The necrotic or ischemic section of the cecum was resected and the abdominal cavity was rinsed with warmed phosphate buffer at $37^{\circ} \mathrm{C}$. Aminoglycoside antibiotics (gentamicin) were administered with a dosage of $6 \mathrm{mg}$ per kilogram intramuscularly. A sterile mesh of $2.5 \times 3 \mathrm{~cm}\left(7.5 \mathrm{~cm}^{2}\right)$ was placed intraperitoneally and was fixated with six transmuscular nonabsorbable sutures (5-0 Ethilon, Ethicon, Inc). Again, the fascia and skin were closed in two layers with a running absorbable suture (5-0 Safil; B. Braun). Subsequently, the rats received $5 \mathrm{~mL}$ sodium chloride 0.9 per cent and were placed under a heating lamp to prevent hypothermia immediately after surgery.

\section{Survival and wellness}

All rats were weighed daily during the first $4 \mathrm{~d}$ postoperatively. Animals were inspected for signs of pain or surgical site occurrences. In addition, all animals were checked daily by an animal care taker. A 12-point wellness and behavior scoring system was used to assess wellness and behavior (Supplementary Materials, Table 1). ${ }^{14}$ Rats were removed from the experiment when they reached the humane endpoint (a wellness score of $<5$ points or weight loss of more than $20 \%$ ).

\section{Sacrifice}

After 30 and $90 \mathrm{~d}$, euthanasia was performed under anesthesia (combination of isoflurane and oxygen inhalation) by subsequent cardiac cut. ${ }^{15}$

\section{Microbiology}

The abdominal skin was shaved and disinfected with alcohol $70 \%$. The ventral abdominal wall was opened via a U-shaped incision, and a picture of the mesh was taken (Figure). Fullthickness abdominal wall samples including mesh were sampled aseptically. The samples measured $1.0 \times 1.0 \mathrm{~cm}$ and

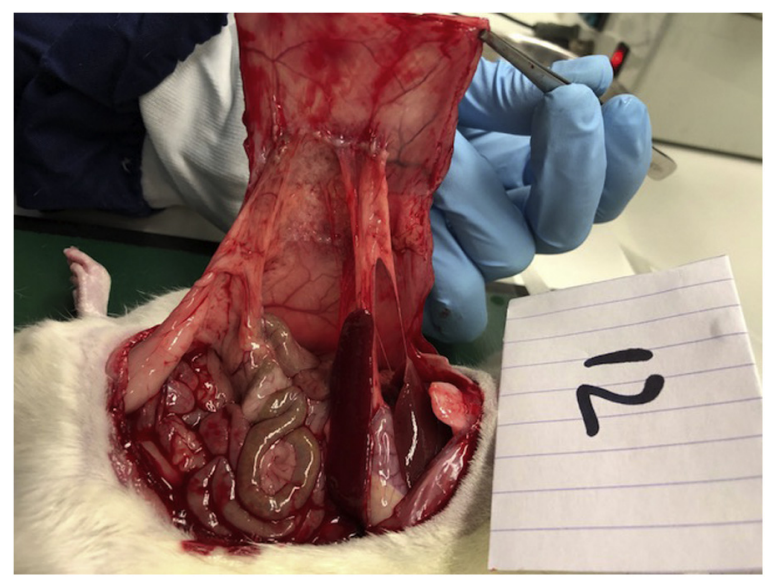

Fig - Photograph (color) taken during the macroscopic assessment. Photo taken during sacrifice showing the inner abdominal wall and a polypropylene mesh without zinc coating. (Color version of figure is available online.) were stored on ice in a tube with $2 \mathrm{~mL}$ sterile phosphate buffered saline. Subsequently, samples were homogenized for $30 \mathrm{~s}$ (IKA T25 ULTRA-TURRAX). Samples were plated in serial dilutions onto MacConkey Agar (Becton Dickinson, Etten-Leur, the Netherlands) to select for gram-negative bacteria. The samples were also plated on trypticase soy agar with $5 \%$ sheep blood (Becton Dickinson) to select for a wide variety of microorganisms. A maximum of three bacteria were identified using the matrix-assisted laser desorption or ionization timeof-flight analyzer (MALDI Biotyper; Bruker Daltonics, Bremen, Germany). The plates were incubated at $37^{\circ} \mathrm{C}$ for $24 \mathrm{~h}$, and the amount of colony forming units (CFU) per full-thickness abdominal wall and mesh sample (CFU/sample) was counted. Second, a qualitative analysis was performed using $30 \mu \mathrm{L}$ inoculation loop. For confirmation of the microbiological flora of healthy Wistar Han rats, additional analyses were performed. Feces from five different healthy Wistar Han rats from the same strain and area (Charles River Laboratories) were collected directly from the cecum and analyzed with the same methods as described previously.

\section{Macroscopy}

All parameters were determined by two blinded, independent observers. In case of disagreement, the results were discussed between the two blinded observers and consensus was reached.

\section{Ingrowth of the mesh}

All edges of the mesh were lifted from the abdominal wall and inspected for ingrowth. Ingrowth was computed by using a caliper to examine adhering tissue between abdominal wall and mesh presented as a percentage. ${ }^{15-17}$

\section{Adhesions}

Adhesions were determined in a qualitative manner by using the Zühlke score (Supplementary Materials, Table 2) and in a quantitative manner by two independent observers until consensus was reached and expressed in percentages on the mesh surface. ${ }^{18}$

\section{Abscesses}

The amount and size of abscesses at the abdominal wall and in the abdominal cavity were assessed visually by using a scoring system (Supplementary Materials, Table 3). ${ }^{19}$

\begin{tabular}{|c|c|c|c|c|c|}
\hline $\begin{array}{l}\text { Mesh } \\
\text { type }\end{array}$ & $\begin{array}{l}\text { Start, } \\
n(\%)\end{array}$ & $\begin{array}{c}\text { Death, } \\
n(\%)\end{array}$ & $\begin{array}{c}\text { Total } \\
\text { FU, } n(\%)\end{array}$ & $\begin{array}{l}\text { 30-day } \\
\text { FU, } n\end{array}$ & $\begin{array}{c}\text { 90-day } \\
\text { FU, } n\end{array}$ \\
\hline ZnMesh & $20(33)$ & $9(45)$ & $11(42)$ & 6 & 5 \\
\hline Control & $18(47)$ & $3(17)$ & $15(58)$ & 6 & 9 \\
\hline Total & $38(100)$ & $12(32)$ & $26(100)$ & 12 & 14 \\
\hline
\end{tabular}

$\mathrm{FU}=$ follow-up 


\section{Table 2 - Cecal ligation puncture model-cecum.}

\begin{tabular}{lc} 
Cecum & $n(\%)$ \\
\hline Necrotic & $16(42.1)$ \\
Ischemic & $15(39.5)$ \\
Ischemic and necrotic (combination) & $1(2.6)$ \\
No changes (normal cecum) & $2(5.3)$ \\
No second operation & $2(5.3)$ \\
Missing & $2(5.3)$ \\
Total & $38(100)$
\end{tabular}

\section{Histology}

Full-thickness (mesh and abdominal wall muscle) samples of $1.0 \times 0.5 \mathrm{~cm}$ were collected in-between sutures. All samples were fixated in $4 \%$ formalin for $24 \mathrm{~h}$. Next, the fixated samples were embedded in paraffin. Sections of $4 \mu \mathrm{m}$ were cut (Leica RM2255 microtome; Leica Biosystems, Wetzlar, Germany) and stained with Sirius Red (Ventana Benchmark Special Stains system; Hoffmann-La Roche, Bazel, Switzerland) or hematoxylin and eosin staining (Ventana Symphony automated staining instrument; Hoffman-La Roche, Bazel, Switserland). All histological evaluations were performed by a pathologist (MCvG) who was blinded for the type of mesh. The inflammatory cell reaction was evaluated by counting the amount of cells per highpower field (40× magnification), using a scoring system described by Peeters et al. (Supplementary Materials, Table 4). ${ }^{20}$ Mesh-specific parameters were evaluated using a modified scoring system assessing scaffold degradation, fibrous encapsulation, cellular infiltration, and neovascularization (Supplementary Materials, Table 5). ${ }^{20}$ Collagen deposition, as visualized by Sirius Red staining, around the mesh and abdominal wall were evaluated using a scoring system described by Deeken et al. (Supplementary Materials,Table 6). ${ }^{21}$

\section{Statistical analysis}

A power calculation was not performed because no earlier comparison in the number of CFU between meshes was performed. Outcomes are presented as median (interquartile range). Survival, macroscopy, histology, and microbiological results were compared performing a $\chi^{2}$ test and a nonparametric Mann-Whitney $U$ test for independent samples. Reported $P$-values are two-sided, and P-values $<0.05$ were considered statistically significant. IBM SPSS Statistics for Windows, version 24.0.0.1, Armonk, NY, was used.

\section{Results}

\section{Survival}

Initially, all rats survived the first operation. In the first $4 \mathrm{~d}$ postoperatively, 12 rats (32\%) of the 38 rats died of sepsis. Nine of 12 rats belonged to the ZnMesh group, and three of 12 rats belonged to the control group. However, two of nine rats from the ZnMesh group had never received a ZnMesh as they died before the second surgery and subsequent mesh implantation. This difference in two groups was not significantly different $(P=0.086)$. One of 12 rats died at day 15 for an unknown reason. None of the rats reached the humane endpoint. Finally, 26 rats (68.5\%) remained for follow-up with 12 rats $(46.2 \%)$ in the 30 -day follow-up group and 14 (53.8\%) in the 90-day follow-up group (Table 1).

\section{Cecal ligation puncture model}

Sixteen rats (42.1\%) had a necrotic cecum and 15 rats (39.5\%) had an ischemic cecum (Table 2). All animals showed symptoms of sepsis, including weight loss, abnormal posture, ocular exudates, apathetic behavior, diarrhea, shivering, and piloerection.

\section{Microbiology}

At $30 \mathrm{~d}$, no significant difference in CFU/sample was present between the ZnMesh and control groups (Table 3). At $90 \mathrm{~d}$, a significantly lower number of CFU/sample were present in the ZnMesh group compared with the control group (0 $\log _{10} \mathrm{CFU} /$ sample, IQR 0-1.40 versus $1.58 \log _{10} \mathrm{CFU} /$ sample IQR 0-4.30, $P=0.012$, Table 3). Mainly, Enterococcus and Staphylococcus, both gram-positive bacteria, were identified. In an additional experiment, mostly Escherichia (a gram-negative bacterium) and Lactobacillus (a gram-positive bacterium) were identified in the feces of five Wistar Han rats. Furthermore, Enterococcus and Staphylococcus were identified.

\section{Macroscopy, ingrowth}

There were no significant differences in ingrowth of the mesh in percentages in both groups at both time points (30 d of

Table 3 - Microbiology, 30 and $90 \mathrm{~d}$ of follow-up.

\begin{tabular}{lccc}
\hline $30 \mathrm{~d}$ of follow-up & ZnMesh $(n=6)$ & Control $(n=6)$ & $P$-value \\
\hline MacConkey $\left(\log _{10}\right.$ CFU/sample) & $3.75(1.11-4.72)$ & $2.93(1.11-5.85)$ & 1.000 \\
TSA-SB $\left(\log _{10}\right.$ CFU/sample) & $3.98(1.94-6.08)$ & $3.98(1.94-6.08)$ & 0.818 \\
\hline $90 \mathrm{~d}$ of follow-up & ZnMesh $(n=5)$ & Control $(n=9)$ & $P$-value \\
\hline MacConkey $\left(\log _{10}\right.$ CFU/sample) & $0(0-2.65)$ & $1.18(0-4.04)$ & 0.438 \\
TSA-SB $\left(\log _{10}\right.$ CFU/sample) & $0(0-1.40)$ & $1.58(0-4.30)$ & 0.012 \\
\hline
\end{tabular}

Statistically significant values $(P<0.05)$ are given in bold.

TSA-SB $=$ trypticase soy agar with $5 \%$ sheep blood. 
Table 4 - Macroscopy: ingrowth and adhesions (\%) 30 and $90 \mathrm{~d}$ of follow-up.

\begin{tabular}{llcc}
\hline 30 d of follow-up & $\begin{array}{l}\text { ZnMesh } \\
(n=6)\end{array}$ & Control $(n=6)$ & P-value \\
\hline Ingrowth (\%) & $75(65-88)$ & $78(70-81)$ & 1.000 \\
\hline Adhesions (\%) & $85(74-96)$ & $75(56-93)$ & 0.394 \\
\hline 90 d of follow-up & $\begin{array}{l}\text { ZnMesh } \\
(n=5)\end{array}$ & Control $(n=9)$ & P-value \\
\hline Ingrowth (\%) & $66(49-74)$ & $59(47-75)$ & 0.797 \\
\hline Adhesions (\%) & $95(60-100)$ & $50(23-75)$ & 0.029 \\
\hline
\end{tabular}

Median (interquartile range).

Statistically significant values $(P<0.05)$ are given in bold.

follow-up: 75 [IQR 65-88] percent versus 78 [IQR 70-81] percent, $P=1.000$; $90 \mathrm{~d}$ of follow-up: 66 [IQR 49-74] percent versus 59 [IQR 47-75] percent, $P=0.797$, see Table 4).

\section{Macroscopy, adhesions}

The highest Zühlke score in the ZnMesh group was Zühlke 3 in six rats (100\%) and Zühlke 3 in five rats (100\%) after 30 and $90 \mathrm{~d}$, respectively. In the control group, the Zühlke score was 3 in four rats (80\%) after $30 \mathrm{~d}$. After $90 \mathrm{~d}$, eight rats (88.9\%) had a Zühlke 3 score. The highest Zühlke score in the control group was Zühlke 4 in two rats (20\%) after $30 \mathrm{~d}$ of follow-up and in one rat (11.1\%) after $90 \mathrm{~d}$ of follow-up. No significant differences were found after $30 \mathrm{~d}$ of follow-up in adhesions expressed in percentage (85 [IQR 74-96] percent versus 75 [IQR 56-93] percent, $P=0.394$, Table 4). The percentage of adhesions on the mesh surface was significantly higher in the ZnMesh group after $90 \mathrm{~d}$ (95 [IQR 60100] versus 50 [IQR 23-75], $P=0.029$, see Table 4).

\section{Macroscopy, abscesses}

Macroscopically, only one rat developed one small abscess located on the mesh. This rat had a regular polypropylene mesh and was randomized for the 90-day follow-up group.

\section{Histology}

Histological analyses showed no significant differences in inflammatory cell reaction (overall inflammatory cell reaction $[P=0.781]$, eosinophils-neutrophils $[P=0.274]$, macrophagesforeign body giant cells $[P=0.432]$, and mononuclear cells $[P=0.432]$, Table 5) and mesh-specific parameters (scaffold degradation $[P=0.820]$, fibrous encapsulation $[P=0.193]$, cellular infiltration $[P=0.595]$, neovascularization $[P=0.820]$, and extracellular matrix deposition $[P=0.820]$, Table 6$)$. In addition, no significant differences were found in collagen deposition across the four groups $(P=0.257$, Table 6 ). Four rats showed microscopically signs of abscess formation, at both time points with one rat implanted with a $\mathrm{ZnMesh}$ and one rat in the control group.

\section{Discussion}

In this rat study, a polypropylene mesh impregnated with zinc ions was compared with a regular polypropylene mesh in a contaminated environment. After a follow-up of $90 \mathrm{~d}$, a lower CFU per sample was found in favor of the ZnMesh on the trypticase soy agar with $5 \%$ sheep blood agar plate. This difference was not seen at the other agar plates after a follow-up of $30 \mathrm{~d}$. In addition, a higher percentage adhesions on the mesh was found in the ZnMesh group after $90 \mathrm{~d}$ of follow-up. Adhesion formation is an important parameter for investigating the biocompatibility of meshes. Prolonged exposure to the mesh and/or the addition of zinc ions could result in more extensive reactions and could be an explanation for this finding. The exact reason for this difference in adhesions between groups remains unclear. No differences were found in macroscopically assessed ingrowth and abscesses between meshes. The histological parameters including inflammatory cell reaction, mesh-specific parameters, and collagen deposition were not significantly different between the two groups after 30 and $90 \mathrm{~d}$. However, the power calculation was not based on these secondary outcomes and might therefore lack enough power to detect a difference.

The mortality after peritonitis induction was $32 \%$, which is slightly higher when compared with previous literature using this cecal ligation puncture model $(10 \%-28 \%)^{13,16,17,22,23} \mathrm{~A}$ notable high mortality rate was seen in the ZnMesh group (nine ZnMesh animals versus three control animals). However, two of these nine rats never received a ZnMesh. These two rats died before implantation due to the implications of the sepsis based on the induced peritonitis. This difference in dead animals between the two groups and mesh types was not significantly different $(P=0.086)$. An explanation for this high mortality could be a less resistant strain of animals for infection or the presence of a more fulminant abdominal infection due to the experimental set-up.

Various meshes are available for the repair of an abdominal wall hernia in the presence of intra-abdominal infection. Still, the introduction of a mesh reduces the amount of bacteria

\section{Table 5 - Histology: inflammatory cell reaction.}

\begin{tabular}{|c|c|c|c|c|c|}
\hline Inflammatory cell reaction & $\begin{array}{c}\text { ZnMesh } \\
(n=6) 30 \mathrm{~d}\end{array}$ & $\begin{array}{c}\text { Control } \\
(n=6) 30 \mathrm{~d}\end{array}$ & $\begin{array}{c}\text { ZnMesh } \\
(n=5) 90 \mathrm{~d}\end{array}$ & $\begin{array}{c}\text { Control } \\
(n=9) 90 \mathrm{~d}\end{array}$ & $P$-value \\
\hline Inflammatory cell reaction & $3(2-3)$ & $3(3-3)$ & $3(2-3)$ & $3(2,3)$ & 0.781 \\
\hline Eosinophils-neutrophils & $3(1-3)$ & $3(3-3)$ & $3(0-3)$ & $2(0-3)$ & 0.274 \\
\hline Macrophages-foreign body giant cells & $3(2-3)$ & $3(2-3)$ & $3(1-3)$ & $3(3-3)$ & 0.432 \\
\hline Mononuclear cells & $3(2-3)$ & $3(2-3)$ & $3(1-3)$ & $2(1-3)$ & 0.432 \\
\hline
\end{tabular}

Median (interquartile range). 
Table 6 - Histology: mesh-specific parameters.

\begin{tabular}{|c|c|c|c|c|c|}
\hline Mesh-specific parameters & $\begin{array}{c}\text { ZnMesh } \\
(n=6) 30 \mathrm{~d}\end{array}$ & $\begin{array}{c}\text { Control } \\
(n=6) 30 \mathrm{~d}\end{array}$ & $\begin{array}{c}\text { ZnMesh } \\
(n=5) 90 \mathrm{~d}\end{array}$ & $\begin{array}{c}\text { Control } \\
(n=9) 90 \mathrm{~d}\end{array}$ & $P$-value \\
\hline Scaffold degradation & $0(0-0)$ & $0(0-0)$ & $0(0-0)$ & $0(0-0)$ & 0.820 \\
\hline Fibrous encapsulation & $1.5(1-2)$ & $1(1-1)$ & $2(1-2)$ & $2(1-2)$ & 0.193 \\
\hline Cellular infiltration & $0(0-0)$ & $0(0-0)$ & $0(0-0)$ & $0(0-1)$ & 0.595 \\
\hline Neovascularization & $0(0-0)$ & $0(0-0)$ & $0(0-0)$ & $0(0-0)$ & 0.820 \\
\hline Extracellular matrix deposition & $0(0-0)$ & $0(0-0)$ & $0(0-0)$ & $0(0-0)$ & 0.820 \\
\hline Collagen deposition & $3.5(2.75-4)$ & $2.5(2-3)$ & $3(2-3.5)$ & $3(2-4)$ & 0.257 \\
\hline
\end{tabular}

Median (interquartile range).

needed to result in an infection by a factor $10^{4}{ }^{24}$ The evidence for using biological mesh in contaminated abdominal wall hernia repair is still limited. ${ }^{25}$ The aim of this experimental study was to add knowledge in this search for an ideal mesh to use in a contaminated environment for ventral hernia repair. The occurrence of a clinically relevant infection depends on both patient-related factors as well as the quantity of bacteria. ${ }^{26}$ An earlier conducted study by Tubre et al. showed that contamination with more than $10^{5} \mathrm{CFU}$ per gram may result in wound infections. ${ }^{26}$ Pathogens found in humans at surgical site infection were S. aureus and Enterococcus species. ${ }^{26}$ These organisms are the same as found in this study, which is performed in rats. Recently, a study showed that rats represent a good preclinical model in hernia and mesh research. ${ }^{27}$ In addition, future studies may consider electron microscopy for the evaluation of biofilm formation because this supports bacterial attachment to the mesh. ${ }^{26}$ The results of this present study may encourage us to conduct more research with zincimpregnated meshes in a contaminated environment, to decrease the risk of surgical site infection or mesh infection after abdominal wall repair. However, a comparison should be made with different types of meshes because the placement of a polypropylene mesh intraperitoneally is certainly not the standard..$^{28}$ New in vitro and in vivo studies could be performed with direct inoculation on the mesh surface with a known quantity and quality of the bacteria, and to compare this with different permanent synthetic, slowly resorbable synthetic and nonsynthetic (biological) meshes.

\section{Limitations}

Information regarding the regular microbiological flora was required to differentiate between contamination during surgery or an effect of the ZnMesh on a fewer amount of CFU per sample in favor of the ZnMesh. However, microbiological assessment of preoperative and intraoperative feces was lacking in this study. Nevertheless, Charles River laboratories kindly provided data regarding the microbiological flora of these rats. These data showed that they found comparable microbiological flora as was found in this present study. Besides, feces from rats from the same laboratory, strain and area were analyzed with the same methods as in this experiment to confirm the additional data from Charles River laboratories. With these supplementary tests, an effect of the ZnMesh on CFU per sample was confirmed. Consensus and comparability among animal experiments to study mesh behavior is lacking. ${ }^{29}$ Several differences between this experimental study and the human situation were present. Examples are the treatment of abdominal sepsis and the relative dimensions of the mesh. ${ }^{15}$ Because this experimental study was performed with animals, these results may not be translated to the human population directly.

\section{Conclusion}

A significantly lower number of CFU per sample were found in the ZnMesh group after $90 \mathrm{~d}$. However, no differences in other outcomes were found between the ZnMesh and control groups after $30 \mathrm{~d}$ of follow-up. These results suggest that a zinc-impregnated mesh has antibacterial properties when placed in a contaminated environment, compared with a regular polypropylene mesh. However, this is at the cost of a significantly higher percentage of adhesions. In addition, an antiadhesive mesh coating could be added to reduce adhesions. Further experiments are required to confirm this hypothesis.

\section{Acknowledgment}

The authors thank T.V.D. van Tienhoven, G. Ambagtsheer, S. van Damme, H. van Willigenburg, and R.W.F. de Bruin for assisting during and after the experiments. The authors thank M. ten Kate for performing and assisting in interpreting microbiological evaluations. The authors would like to remember our late microbiologist: Professor J.W. Mouton.

The regular polypropylene and ZnMesh was provided free of charge by Parx Plastics, Rotterdam, the Netherlands. Parx Plastics was not involved in the design or conduct of the study, analysis of the results, or preparation of the manuscript.

Author contributions: Y. Yurtkap Data curation; formal analysis; project administration; writing - review and editing; A.P. Jairam Conceptualization; investigation; methodology; validation; writing - review and editing; R. Kaufmann Conceptualization; funding acquisition; investigation; methodology; validation; writing - review and editing; L.F. Kroese Conceptualization; investigation; methodology; validation; writing - review and editing; M.C. Clahsen-van Groningen 
Investigation; supervision; validation; writing - review and editing; J.W. Mouton Investigation; supervision; validation; writing - review and editing; A.G. Menon Conceptualization; supervision; validation; writing - review and editing; G.J. Kleinrensink Conceptualization; supervision; validation; writing - review and editing; J.J. Jeekel Conceptualization; supervision; validation; writing - review and editing; J.F. Lange Data curation; conceptualization; funding acquisition; supervision; writing - review and editing; E.J. Belt Conceptualization; funding acquisition; supervision; writing - review and editing.

\section{Disclosure}

The authors report no proprietary or commercial interest in any product mentioned or concept discussed in this article.

\section{Supplementary data}

Supplementary data related to this article can be found at https://doi.org/10.1016/j.jss.2019.09.046.

\section{R E F E R E N C E S}

1. Burger JW, Luijendijk RW, Hop WC, Halm JA, Verdaasdonk EG, Jeekel J. Long-term follow-up of a randomized controlled trial of suture versus mesh repair of incisional hernia. Ann Surg. 2004;240:578-583. discussion 83-5.

2. Rosen MJ, Bauer JJ, Harmaty M, et al. Multicenter, prospective, longitudinal study of the recurrence, surgical site infection, and quality of life after contaminated ventral hernia repair using biosynthetic absorbable mesh: the COBRA study. Ann Surg. 2017;265:205-211.

3. Krpata DM, Blatnik JA, Novitsky YW, Rosen MJ. Evaluation of high-risk, comorbid patients undergoing open ventral hernia repair with synthetic mesh. Surgery. 2013;153:120-125.

4. Bueno-Lledo J, Torregrosa-Gallud A, Sala-Hernandez A, et al. Predictors of mesh infection and explantation after abdominal wall hernia repair. Am J Surg. 2017;213:50-57.

5. Madani A, Niculiseanu P, Marini W, et al. Biologic mesh for repair of ventral hernias in contaminated fields: long-term clinical and patient-reported outcomes. Surg Endosc. 2017;31:861-871.

6. Rosen MJ, Krpata DM, Ermlich B, Blatnik JA. A 5-year clinical experience with single-staged repairs of infected and contaminated abdominal wall defects utilizing biologic mesh. Ann Surg. 2013;257:991-996.

7. Reynolds D, Davenport DL, Korosec RL, Roth JS. Financial implications of ventral hernia repair: a hospital cost analysis. J Gastrointest Surg. 2013;17:159-166. discussion p.66-67.

8. Blatnik JA, Thatiparti TR, Krpata DM, Zuckerman ST, Rosen MJ, von Recum HA. Infection prevention using affinity polymer-coated, synthetic meshes in a pig hernia model. J Surg Res. 2017;219:5-10.

9. Perez-Kohler B, Benito-Martinez S, Rodriguez M, GarciaMoreno F, Pascual G, Bellon JM. Experimental study on the use of a chlorhexidine-loaded carboxymethylcellulose gel as antibacterial coating for hernia repair meshes. Hernia. 2019;23:789-800.
10. Klinge U, Junge K, Spellerberg B, Piroth C, Klosterhalfen B, Schumpelick V. Do multifilament alloplastic meshes increase the infection rate? Analysis of the polymeric surface, the bacteria adherence, and the in vivo consequences in a rat model. J Biomed Mater Res. 2002;63:765-771.

11. Huang P, Ma K, Cai X, et al. Enhanced antibacterial activity and biocompatibility of zinc-incorporated organic-inorganic nanocomposite coatings via electrophoretic deposition. Colloids Surf B Biointerfaces. 2017;160:628-638.

12. Kilkenny C, Browne WJ, Cuthill IC, Emerson M, Altman DG. Improving bioscience research reporting: the ARRIVE guidelines for reporting animal research. PLoS Biol. 2010;8:e1000412.

13. Deerenberg EB, Mulder IM, Ditzel M, et al. Polyvinyl alcohol hydrogel decreases formation of adhesions in a rat model of peritonitis. Surg Infect (Larchmt). 2012;13:321-325.

14. Komen N, van der Wal HC, Ditzel M, Kleinrensink GJ, Jeekel H, Lange JF. Colorectal anastomotic leakage: a new experimental model. J Surg Res. 2009;155:7-12.

15. Kaufmann R, Jairam AP, Mulder IM, et al. Characteristics of different mesh types for abdominal wall repair in an experimental model of peritonitis. Br J Surg. 2017;104:1884-1893.

16. Deerenberg EB, Mulder IM, Grotenhuis N, Ditzel M, Jeekel J, Lange JF. Experimental study on synthetic and biological mesh implantation in a contaminated environment. Br J Surg. 2012;99:1734-1741.

17. Mulder IM, Deerenberg EB, Bemelman WA, Jeekel J, Lange JF. Infection susceptibility of crosslinked and non-crosslinked biological meshes in an experimental contaminated environment. Am J Surg. 2015;210:159-166.

18. Zuhlke HV, Lorenz EM, Straub EM, Savvas V. [Pathophysiology and classification of adhesions] Pathophysiologie und Klassifikation von Adhasionen. Langenbecks Arch Chir Suppl II Verh Dtsch Ges Chir. 1990:1009-1016.

19. Rodgers KE, Schwartz HE, Roda N, Thornton M, Kobak W, diZerega GS. Effect of oxiplex* films (PEO/CMC) on adhesion formation and reformation in rabbit models and on peritoneal infection in a rat model. Fertil Steril. 2000;73:831-838.

20. Peeters E, van Barneveld KW, Schreinemacher MH, et al. Oneyear outcome of biological and synthetic bioabsorbable meshes for augmentation of large abdominal wall defects in a rabbit model. J Surg Res. 2013;180:274-283.

21. Deeken CR, Matthews BD. Ventralight ST and SorbaFix versus physiomesh and securestrap in a porcine model. JSLS. 2013;17:549-559.

22. Wu Z, Vakalopoulos KA, Boersema GS, et al. The prevention of colorectal anastomotic leakage with tissue adhesives in a contaminated environment is associated with the presence of anti-inflammatory macrophages. Int J Colorectal Dis. 2014;29:1507-1516.

23. Reijnen MM, Skrabut EM, Postma VA, Burns JW, van Goor H. Polyanionic polysaccharides reduce intra-abdominal adhesion and abscess formation in a rat peritonitis model. J Surg Res. 2001;101:248-253.

24. Halaweish I, Harth K, Broome AM, Voskerician G, Jacobs MR, Rosen MJ. Novel in vitro model for assessing susceptibility of synthetic hernia repair meshes to Staphylococcus aureus infection using green fluorescent protein-labeled bacteria and modern imaging techniques. Surg Infect (Larchmt). 2010;11:449-454.

25. Kamarajah SK, Chapman SJ, Glasbey J, et al. Systematic review of the stage of innovation of biological mesh for complex or contaminated abdominal wall closure. BJS Open. 2018;2:371-380. 
26. Tubre DJ, Schroeder AD, Estes J, Eisenga J, Fitzgibbons Jr RJ. Surgical site infection: the "Achilles Heel" of all types of abdominal wall hernia reconstruction. Hernia. 2018;22:1003-1013.

27. van den Hil LCL, Vogels RRM, van Barneveld KWY, et al. Comparability of histological outcomes in rats and humans in a hernia model. J Surg Res. 2018;229:271-276.
28. Halm JA, de Wall LL, Steyerberg EW, Jeekel J, Lange JF. Intraperitoneal polypropylene mesh hernia repair complicates subsequent abdominal surgery. World J Surg. 2007;31:423-429. discussion 30.

29. Vogels RRM, Kaufmann R, van den Hil LCL, et al. Critical overview of all available animal models for abdominal wall hernia research. Hernia. 2017;21:667-675. 